\title{
Low-grade glioma surgery in eloquent areas: volumetric analysis of extent of resection and its impact on overall survival. A single-institution experience in 190 patients
}

\author{
Clinical article
}

\author{
Tamara Ius, M.D., ${ }^{1,2}$ Miriam Isola, Ph.D., ${ }^{3}$ Riccardo Budai, M.D. ${ }^{4}$ Giada Pauletto, M.D., Ph.D., ${ }^{4}$ \\ Barbara Tomasino, Ph.D., ${ }^{5}$ Luciano Fadiga, M.D., Ph.D., ${ }^{2,6}$ and Miran Skrap, M.D. ${ }^{1}$ \\ Departments of ${ }^{I}$ Neurosurgery and ${ }^{4}$ Neurology, Azienda Ospedaliero-Universitaria Santa Maria della Misericordia, \\ Udine; ${ }^{3}$ Department of Medical and Biological Sciences, Section of Statistics, University of Udine; ${ }^{2}$ Department of \\ Robotics, Brain and Cognitive Sciences, Instituto Italiano di Tecnologia, Genoa; and IRCCS "E. Medea," Polo \\ Regionale del FVG, San Vito al Tagliamento, Pordenone; and ${ }^{6}$ Section of Human Physiology, University of Ferrara, Italy
}

Object. A growing number of published studies have recently demonstrated the role of resection in overall survival (OS) for patients with gliomas. In this retrospective study, the authors objectively investigated the role of the extent of resection (EOR) in OS in patients with low-grade gliomas (LGGs).

Methods. Between 1998 and 2011, 190 patients underwent surgery for LGGs. All surgical procedures were conducted under corticosubcortical stimulation. The EOR was established by analyzing the pre- and postoperative volumes of the gliomas on T2weighted MRI studies. The difference between the preoperative tumor volumes was also investigated by measuring the volumetric difference between the T2- and T1-weighted MRI images ( $\triangle$ VT2T1) to evaluate how the diffusive tumor-growing pattern affected the EOR achieved.

Results. The median preoperative tumor volume was $55 \mathrm{~cm}^{3}$, and in almost half of the patients the EOR was greater than $90 \%$. In this study, patients with an EOR of $90 \%$ or greater had an estimated 5-year OS rate of 93\%, those with EOR between $70 \%$ and $89 \%$ had a 5 -year OS rate of $84 \%$, and those with EOR less than $70 \%$ had a 5 -year OS rate of $41 \%(\mathrm{p}<0.001)$. New postoperative deficits were noted in $43.7 \%$ of cases, while permanent deficits occurred in $3.16 \%$ of cases. There were 41 deaths (21.6\%), and the median follow-up was 4.7 years.

A further volumetric analysis was also conducted to compare 2 different intraoperative protocols (Series 1 [intraoperative electrical stimulation alone] vs Series 2 [intraoperative stimulation plus overlap of functional MRI/fiber tracking diffusion tensor imaging data on a neuronavigation system]). Patients in Series 1 had a median EOR of $77 \%$, while those in Series 2 had a median EOR of $90 \%(\mathrm{p}=0.0001)$. Multivariate analysis showed that OS is influenced not only by EOR $(\mathrm{p}=0.001)$ but also by age $(\mathrm{p}=$ $0.003)$, histological subtype $(\mathrm{p}=0.005)$, and the $\Delta$ VT2T1 value $(\mathrm{p}<0.0001)$. Progression-free survival is similarly influenced by histological subtype (fibrillary astrocytoma, $\mathrm{p}=0.003)$, EOR $(\mathrm{p}<0.0001)$, and $\Delta$ VT2T1 value $(\mathrm{p}<0.0001)$, as is malignant progression-free survival $(\mathrm{p}=0.003, \mathrm{p}<0.0001$, and $\mathrm{p}<0.0001$, respectively). Finally, the study shows that the higher the $\Delta V \mathrm{VT} 2 \mathrm{~T}$ value, the less extensive the currently possible resection, highlighting an apparent correlation between the $\Delta$ VT2T1 value itself and EOR ( $<<0.0001)$.

Conclusions. The EOR and the $\triangle \mathrm{VT} 2 \mathrm{~T} 1$ values are the strongest independent predictors in improving OS as well as in delaying tumor progression and malignant transformation. Furthermore, the $\Delta$ VT2T1 value may be useful as a predictive index for EOR. Finally, due to intraoperative corticosubcortical mapping and the overlap of functional data on the neuronavigation system, major resection is possible with an acceptable risk and a significant increase in expected OS.

(http://thejns.org/doi/abs/10.3171/2012.8.JNS12393)

\section{KEY WoRDS • low-grade glioma • brain mapping • extent of tumor resection direct electrical stimulation $\bullet$ tumor volume - survival $\bullet$ oncology}

$\mathrm{D}$ IFFUSE infiltrative LGGs of the cerebral hemispheres in adults represent up to $30 \%$ of gliomas. ${ }^{20}$ Despite an initial indolent subclinical growth rate,

Abbreviations used in this paper: $\Delta \mathrm{VT} 2 \mathrm{~T} 1=$ volumetric difference between preoperative tumor volumes on T2- and T1-weighted MRI studies; DTI = diffusion tensor imaging; EBRT = externalbeam radiation therapy; $\mathrm{EOR}=$ extent of resection; $\mathrm{fMRI}=$ functional MRI; HR = hazard ratio; ICC = intraclass correlation coefficient; IES = intraoperative electrical stimulation; KPS $=$ Karnofsky Performance Scale; LGG = low-grade glioma; $\mathrm{LOH}=$ loss of heterozygosity; MPFS = malignant progression-free survival; OS $=$ overall survival; $\mathrm{PFS}=$ progression-free survival. the normal pattern is for continuous growth and systematic progression to malignant transformation, in turn leading to neurological disability and ultimately to death. ${ }^{19,55}$

The most critical factor in achieving a radical resection in LGGs is their involvement of eloquent cortical areas and subcortical functional pathways..$^{20,34}$ Consequently, for many years, the role of surgery in LGG management has been a matter of debate. ${ }^{7,23,54}$

Recently, a large number of surgical studies based on the objective evaluation of EOR have been published, suggesting that EOR has a significant effect not only on the rate of tumor progression and OS but also on the decrease 
in risk of anaplastic transformation. ${ }^{28,35,36,46-49,54}$ Moreover, it is generally thought that an extensive resection, performed at the time of initial diagnosis, is the greatest factor for improving patient OS. $35,36,49,52$

However, no randomized trials have specifically addressed the effect of EOR on OS, PFS, and MPFS among patients with LGGs. Most studies since 1990 have provided a subjective analysis, based on the surgeon's intraoperative impression, rather than a statistical analysis to examine the efficacy of EOR in improving patient sur-

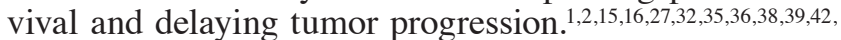
43,49,52,54,58,60,61 Only 5 studies have included a volumetric analysis of EOR, multivariate, and univariate analyses, to determine the statistical significance of EOR. ${ }^{16,49,52,54,58}$ Of these, the largest analysis with volumetric quantification of EOR demonstrated a major effect of EOR on OS for hemispheric LGGs. Indeed, for patients who undergo gross-total resection, the 10-year survival rate approaches $100 \%$, while the survival rate progressively decreases as the EOR approaches $40 \%{ }^{48}$ Consequently, despite the lack of Class I evidence, ${ }^{28,46,47,54}$ surgical treatment has recently been identified as the first option in the management of LGGs. ${ }^{55}$

A prospective study evaluating patients with LGGs is highly unlikely to be conducted; therefore, as a more practical way to address the lack of Class I evidence, a randomized study with prospective observations was conducted to better define the role of EOR of LGGs and to confirm the value of the few volumetric studies published to date.

Although EOR has been statistically proven to be the most significant independent factor for OS, preservation of patients' simple and complex functions is clearly mandatory. $3,7,10,16,18,45,54$ For these reasons, at our institution the first option in the treatment of LGGs is resection performed using intraoperative brain mapping techniques. This allows maximization of tumor resection while minimizing postoperative morbidity. $4,7,8,11,18,21,22,54$ In this study, we present our paradigm for the surgical treatment of LGGs, focusing on the effect of EOR on OS, PFS, and MPFS.

Finally, the $\Delta \mathrm{VT} 2 \mathrm{~T} 1$ value, a preoperative estimation of the difference between tumor volumes on $\mathrm{T} 2$-weighted MRI studies and on contrast-enhanced T1-weighted MRI studies, has been proposed as a predictive analytical index of EOR to discriminate between the proliferative growth mechanism and the diffusive spreading mechanism along the white matter in LGG development. ${ }^{52}$ Therefore, on the basis of pre- and postoperative MRI volumetric tumor analyses, we also investigated the correlation between this neuroradiological preoperative index and the degree of resection achieved.

\section{Methods}

\section{Study Population}

We analyzed 190 patients who underwent resection for supratentorial LGGs between June 1998 and May 2011. All surgical procedures were performed by the senior author (M.S.). Clinical, radiological, and operative data and hospital course records were reviewed retrospectively.

Preoperatively, all patients underwent neuropsychological evaluation and fMRI for motor and language functions. In addition, language handedness dominance was evaluated using the Edinburgh Inventory Questionnaire. ${ }^{41}$ One hundred twenty-six patients had a lesion involving the dominant hemisphere. No patient had enhancement on contrast-administered T1-weighted MRI sequences. Histological type was determined according to the WHO brain tumor classification..$^{33}$ To create a more uniform study population, patients with gemistocytic histology were excluded, as were patients with foci of anaplastic transformation present in the histological analysis.

Follow-up evaluation was uniform in all patients; that is, neurological examination and control MRI studies were obtained at regular intervals. This study was approved by the local institutional ethics committee on human research.

\section{Surgical Technique and Brain Mapping}

All 190 patients had a tumor involving eloquent areas. The surgical procedures were conducted under cortical and subcortical white matter IES, according to the intraoperative technique previously described by Berger and based on the methodology of Ojemann and Berger. ${ }^{5,6,40} \mathrm{~A}$ neuronavigation system (StealthStation, Medtronic) was used in all cases.

Awake surgery was performed in $66.3 \%$ of patients. The 126 patients with lesions located in the somatosensory area and in the dominant temporal lobe, premotor lobe, insula, and inferior parietal lobe underwent surgery after administration of local anesthesia while intraoperative sensorimotor and language mapping were performed. Conversely, the 64 patients harboring lesions involving nondominant supplementary and primary motor areas and nondominant insular lobes underwent surgery after induction of general anesthesia, in which cortical and subcortical IES enabled the detection of corticospinal pathways.

A standard anesthesiology protocol was used for the surgical procedure, with all patients remaining awake from the beginning of the procedure with light sedation during skull opening and closing. Electroencephalography and electrocorticography recordings were obtained throughout the procedure to monitor the occurrence of afterdischarge phenomena and intraoperative seizures, both electrical and clinical. Motor evoked potential and somatosensory evoked potential recordings were also made throughout the duration of surgery with the aid of an Eclipse Neurological Workstation (Axon Systems, Inc.) and Brain Quick Plus (Micromed, Inc.).

In performing the cortical and subcortical brain mapping, a maximum of $4 \mathrm{~mA}$ of current intensity was generally sufficient for the cortex, and 6 or $8 \mathrm{~mA}$ was sufficient for subcortical simulation. As the surgery proceeded, resection of thin tumor layers was alternated with subcortical IES to detect the functional subcortical motor and language pathways, in accordance with previous technical surgical glioma studies. ${ }^{17,22}$ Standard language tests such as counting, picture naming, and reading tasks 
were systematically performed to determine the essential cortical language sites. More recently, in addition to the IES and standard language tasks, enriched and continuous testing was introduced throughout the procedure, providing complementary information to that provided by the IES.

In the present study, we analyzed a population of patients who underwent surgery for LGG in eloquent areas between June 1998 and May 2011, during which the intraoperative technical protocol was changed. Therefore, 2 consecutive periods were identified to evaluate the impact of each protocol on EOR. In Series 1 (June 1998-December 2004), 73 patients underwent surgery with the aid of corticosubcortical IES, neurophysiological monitoring, and intraoperative use of the neuronavigation system. In Series 2 (January 2005-May 2011), 117 patients underwent surgery using the same intraoperative protocol used in Series 1 but with the addition of overlapping fMRI/ DTI data on the T1-weighted/T2-weighted 3D MRI studies to plan the surgery more precisely.

\section{Patient Outcome Measurements}

Postoperative functional outcome was assessed using the same protocol as before surgery, during the immediate postoperative stage, and at 3 and 6 months after surgery. In addition to neurological morbidity, OS, PFS, and MPFS were assessed. Overall survival was defined as the time between initial surgery and death. Progression-free survival was defined as the time between the initial surgery and demonstration of unequivocal increase in tumor size as shown on follow-up images, malignant progression, or death. Malignant PFS was defined as the time between initial surgery and demonstration of $\mathrm{Gd}$ enhancement on follow-up imaging and/or higher-grade tumor identification from a subsequent biopsy, or death. ${ }^{54}$ Patients with no clinical improvement at the 6-month follow-up examination were considered to have a permanent deficit.

\section{Volumetric Analysis}

Magnetic resonance images in DICOM format were used to assess both pre- and postoperative tumor volume by using axial T2-weighted MRI studies. All preand postoperative tumor segmentations were performed manually across all MRI slices using the OsiriX software tool. ${ }^{24,44,52}$ After segmentation, the volumes were calculated in cubic centimeters. The postoperative volume was measured using images acquired 4 months after surgery.

To avoid subjective bias in manual segmentation for tumor volumes, the preoperative volumetric analysis (computed on T2- and T1-weighted MRI studies) and postoperative volumetric analysis (computed on T2-weighted MRI studies) were performed in a doubleblinded manner by 2 authors (T.I. and M.S.). No discrepancies regarding the tumor volume computed on T2- and T1-weighted MRI studies were found. The interobserver agreement for preoperative tumor volume on T1- and T2weighted images and postoperative tumor volume on $\mathrm{T} 2$ weighted images was expressed as the ICC.

The EOR was calculated as follows: (preoperative tu- mor volume - postoperative tumor volume)/preoperative tumor volume, as previously described by Smith et al..$^{54}$ Finally, with the aim of evaluating the role of the diffusive tumor growth pattern on OS, PFS, and MPFS, and using the methodological procedure described by Skrap et al.,52 we also assessed the volumetric preoperative tumoral difference on T2- and T1-weighted MRI studies for each case as follows: $\Delta \mathrm{VT} 2 \mathrm{~T} 1$ value $=$ preoperative volumetric tumor volume segmented on T2-weighted images - preoperative volumetric tumor volume segmented on T1-weighted images.

\section{Statistical Analysis}

General standard statistical measures were calculated as follows. Characteristics of the study population are described using the median and range for continuous variables and percentages for categorical variables. Data were tested for normal distribution using the Kolmogorov-Smirnov test. The t-test or Mann-Whitney test, as appropriate, was used to compare continuous variables. For categorical variables, cross-tabulations were generated, and a chi-square or Fisher exact test was used to compare distributions. Interobserver reproducibility of tumor volumes was assessed using the ICC and 95\% CI.

For OS, PFS, and MPFS, the Kaplan-Meier approach was used. Patients with no known progression, malignant or otherwise, were censored as of their last scan date. Analysis of survival was done using Cox proportional hazard models, after the proportional hazards assumption had been verified.

In univariate analysis, variables considered as possible prognostic factors were age, sex, KPS score, preoperative tumor volume, tumor histological subtype, tumor location, tumor side, intraoperative protocol used, EOR, residual tumor volume, $\Delta$ VT2T1 value, chemotherapy, and EBRT performed at tumor recurrence or malignant transformation.

The EOR was modeled both as a continuous and an ordinal variable $(<70 \%, 70 \%-89 \%$, and $\geq 90 \%)$ in univariate analysis to ensure consistency with previous studies that focused on the impact of glioma resection in terms of volumes. ${ }^{49,52,54}$

The $\Delta$ VT2T1 value and residual tumor volumes were similarly treated as both continuous and ordinal variables. The $\Delta$ VT2T1 interval was either smaller than $30 \mathrm{~cm}^{3}$ or was $30 \mathrm{~cm}^{3}$ or more, and the residual tumor volume was subdivided into the following 4 groups: smaller than 10 $\mathrm{cm}^{3}, 10-19 \mathrm{~cm}^{3}, 20-29 \mathrm{~cm}^{3}$, and $30 \mathrm{~cm}^{3}$ or larger.

Preoperative tumor volume was treated as a continuous variable, and a log transformation (base 10) was performed to help prevent inappropriate weighting of the results by tumors with large volumes. Multivariate stepwise backward analyses included all variables significant at $p$ $\leq 0.15$ in univariate analysis. Retention in the stepwise model required the variable to be significant at $\mathrm{p}<0.05$ in a multivariate analysis. ${ }^{57}$

Results are presented as HRs and 95\% CIs. Parametric or nonparametric correlation analysis, as appropriate, was used to explore a possible association between differences in preoperative tumor volumes computed on T2-weighted MRI studies, $\Delta \mathrm{VT} 2 \mathrm{~T} 1$ value, and EOR 
achieved. All analyses were conducted using Stata/SE (version 12.0 Stata Corp.) for Microsoft Windows. All 2 -tailed statistical significance levels were set at $\mathrm{p}<0.05$.

\section{Results}

\section{Study Population}

Patient demographic, preoperative clinical, and radiological data are listed in Table 1. All lesions were near TABLE 1: Summary of preoperative clinical and radiological
features in 190 patients with LGGs in eloquent areas

\begin{tabular}{|c|c|}
\hline Parameter & Value $^{*}$ \\
\hline \multicolumn{2}{|l|}{ sex } \\
\hline male & $111(58.4)$ \\
\hline female & $79(41.6)$ \\
\hline \multicolumn{2}{|l|}{ age at diagnosis in yrs } \\
\hline$<40$ & $119(62.6)$ \\
\hline $40-60$ & $58(30.5)$ \\
\hline$>60$ & $13(6.8)$ \\
\hline median age at diagnosis in yrs & $37(18-75)$ \\
\hline \multicolumn{2}{|l|}{ symptoms at presentation } \\
\hline seizures of recent onset & $171(90)$ \\
\hline generalized & $107(56.3)$ \\
\hline partial & $64(33.7)$ \\
\hline incidental & $5(2.6)$ \\
\hline headache & $6(3.2)$ \\
\hline neurological deficits & $8(4.2)$ \\
\hline \multicolumn{2}{|l|}{ preop KPS score } \\
\hline 80 & $5(2.6)$ \\
\hline 90 & $52(27.4)$ \\
\hline 100 & $133(70)$ \\
\hline median preop KPS score & $96.7(80-100)$ \\
\hline \multicolumn{2}{|l|}{ tumor location } \\
\hline precentral area & $83(43.7)$ \\
\hline insula & $56(29.5)$ \\
\hline temporal lobe & $30(15.8)$ \\
\hline postcentral area & $21(11.1)$ \\
\hline \multicolumn{2}{|l|}{ side of tumor } \\
\hline It & $106(55.8)$ \\
\hline $\mathrm{rt}$ & $84(44.2)$ \\
\hline \multicolumn{2}{|l|}{ preop tumol vol on T2-weighted MRI in $\mathrm{cm}^{3}$} \\
\hline$<25$ & $41(21.6)$ \\
\hline $25-50$ & $51(26.8)$ \\
\hline $51-100$ & $47(24.7)$ \\
\hline $100-250$ & $51(26.8)$ \\
\hline median tumor vol on T2-weighted MRI in $\mathrm{cm}^{3}$ & $55(4-260)$ \\
\hline \multicolumn{2}{|l|}{$\Delta \mathrm{VT} 2 \mathrm{~T} 1 \mathrm{in} \mathrm{cm}^{3}$} \\
\hline$<30$ & $116(61)$ \\
\hline$\geq 30$ & $74(39)$ \\
\hline
\end{tabular}

* Median values are presented as the median (range). All other values represent the number of patients (\%). or within the so-called eloquent areas. Motor and speech mapping were performed in $136(71.6 \%)$ and $126(66.3 \%)$ patients, respectively. Symptoms included seizures in 171 patients, headaches in 6 patients, and neurological deficit of recent onset in 8 patients. In 5 patients the tumor diagnosis was incidental to other investigations. None of the patients in this series underwent preoperative chemotherapy or radiotherapy. In all cases, preoperative MRI studies showed a lesion that was hypointense on a T1weighted MRI sequence without Gd contrast enhancement and hyperintense on a T2-weighted MRI sequence. The median preoperative tumor volume, measured on T2weighted images, was $55 \mathrm{~cm}^{3}$ (range $4-260 \mathrm{~cm}^{3}$ ).

\section{Postoperative Course}

The clinical, histological, radiological, and followup data are summarized in Table 2. In summary, new postoperative deficits were noted in 83 patients (43.7\%). At the 6-month follow-up examination, all but 6 patients (3.16\%) improved and returned to their initial level or better. Moreover, the 8 patients with preoperative neurological deficits completely recovered 3 months after surgery. Results of the neuropathological examination revealed a WHO Grade II glioma in all cases. Tumor diagnosis was as follows: 98 fibrillary astrocytomas, 58 oligodendrogliomas, and 34 mixed oligoastrocytomas.

Radiotherapy alone (in 43 patients) or together with chemotherapy (in 27 patients) was administered when tumor resection was less than $70 \%$ or at malignant tumor transformation. Chemotherapy alone was administered when tumor progression was noted on imaging (20 patients). A detailed description of these data is beyond the scope of this study.

\section{Volumetric Analysis}

The interobserver agreement for preoperative tumor volume on T1- and T2-weighted images, as well as the measurement of postoperative tumor volume on T2weighted images, was expressed as the ICC.

The intraobserver agreement was high for all volumetric analyses performed. In detail, the ICC was 0.999 (95\% CI 0.999-0.999), 0.999 (95\% CI 0.999-0.999), and 0.996 (95\% CI 0.994-0.997) for preoperative tumor volume segmented on T1- and T2-weighted MRI studies and postoperative tumor volume computed on T2-weighted MRI studies, respectively.

Data on tumor resections are shown in Table 2. The median residual tumor volume, computed on postoperative T2-weighted MRI studies, was $7 \mathrm{~cm}^{3}$ (range $0-125$ $\mathrm{cm}^{3}$ ). Notably, in almost half of the patients, the EOR was greater than $90 \%$. In particular, the median EOR was $87.5 \%$ (range $28 \%-100 \%$ ). The impact of each intraoperative protocol used on EOR achieved was also evaluated by separating the study population based on the intraoperative protocol adopted (Series 1 vs Series 2). Patients in Series 1 had a mean EOR of $77 \%$ (range $28 \%-100 \%$ ), while patients in Series 2 had a mean EOR of $90 \%$ (range 49\%-100\%) (Mann-Whitney test, z $-4.99 ; \mathrm{p}<0.0001$ ) (Fig. 1). Finally, the effect of the preoperative $\Delta V \mathrm{VT} 2 \mathrm{~T} 1$ value on EOR achieved was investigated. 
TABLE 2: Summary of postoperative clinical findings, histological results, and volumetric analysis of EOR*

\begin{tabular}{|c|c|}
\hline Parameter & Value \\
\hline \multicolumn{2}{|l|}{ immediate postop clinical findings } \\
\hline no deficits & $107(56.3)$ \\
\hline neurological deficits & $83(43.7)$ \\
\hline motor deficits & $48(25.3)$ \\
\hline SMA syndrome & $10(5.3)$ \\
\hline facial droop & $7(3.7)$ \\
\hline UL/LL paresis & $14(7.4)$ \\
\hline UL/LL weakness & $18(9.5)$ \\
\hline speech disorders & $29(15.3)$ \\
\hline expressive aphasia & $21(11.1)$ \\
\hline dysarthria & $8(4.2)$ \\
\hline partial Gerstmann syndrome & $1(0.5)$ \\
\hline sensory UL \&/or LL deficit & $4(2.1)$ \\
\hline postop EDH† & $1(0.5)$ \\
\hline \multicolumn{2}{|l|}{ clinical outcome at 6 mos } \\
\hline no deficits & $184(96.8)$ \\
\hline neurological deficits & $6(3.16)$ \\
\hline motor deficits & $2(1.05)$ \\
\hline SMA syndrome & $0(0.0)$ \\
\hline facial droop & $0(0.0)$ \\
\hline UL/LL paresis & $2(1.05)$ \\
\hline UL/LL weakness & $0(0.0)$ \\
\hline speech disorders & $2(1.05)$ \\
\hline expressive aphasia & $2(1.05)$ \\
\hline dysarthria & $0(0.0)$ \\
\hline partial Gerstmann syndrome & $0(0.0)$ \\
\hline UL \&/or LL sensory deficit & $2(1.05)$ \\
\hline \multicolumn{2}{|l|}{ diagnosis (WHO Grade II) } \\
\hline fibrillary astrocytoma & $98(51.6)$ \\
\hline oligoastrocytoma & $34(17.9)$ \\
\hline oligodendroglioma & $58(30.5)$ \\
\hline \multicolumn{2}{|l|}{$\%$ EOR $\ddagger$} \\
\hline$\geq 90$ & $91(47.9)$ \\
\hline $70-89$ & $69(36.3)$ \\
\hline$<70$ & $30(15.8)$ \\
\hline median $\%$ EOR & $87.5(28-100)$ \\
\hline \multicolumn{2}{|l|}{ Series 1 (IES) } \\
\hline no. of patients & $73(38.4)$ \\
\hline median \% EOR & $77.4(28-100)$ \\
\hline \multicolumn{2}{|l|}{ Series 2 (IES +fMRI/DTI overlap) } \\
\hline no. of patients & $117(61.6)$ \\
\hline median \% EOR & $90(49-100)$ \\
\hline \multicolumn{2}{|l|}{ Subgroup $\mathrm{A}\left(\Delta \mathrm{VT} 2 \mathrm{~T} 1<30 \mathrm{~cm}^{3}\right)$} \\
\hline no. of patients & $116(61.1)$ \\
\hline median $\%$ EOR & $91(49-100)$ \\
\hline
\end{tabular}

(continued)
TABLE 2: Summary of postoperative clinical findings, histological results, and volumetric analysis of EOR* (continued)

\begin{tabular}{|c|c|}
\hline Parameter & Value \\
\hline \multicolumn{2}{|l|}{ Subgroup $B\left(\Delta V T 2 T 1 \geq 30 \mathrm{~cm}^{3}\right)$} \\
\hline no. of patients & $74(38.9)$ \\
\hline median \% EOR & $79(28-100)$ \\
\hline \multicolumn{2}{|l|}{ postop tumor vol in $\mathrm{cm}^{3}$} \\
\hline$<10$ & $111(58.4)$ \\
\hline $11-20$ & $41(21.6)$ \\
\hline $21-30$ & $16(8.4)$ \\
\hline$\geq 30$ & $22(11.6)$ \\
\hline median postop tumor vol in $\mathrm{cm}^{3}$ & $7(0.00-125)$ \\
\hline \multicolumn{2}{|l|}{ clinical follow-up§ } \\
\hline median follow-up in mos & $57(4-155)$ \\
\hline no. of patient deaths & $41(21.6)$ \\
\hline no. w/ disease progression & $79(41.6)$ \\
\hline median time to progression & 3.5 yrs (4-155 mos) \\
\hline no. w/ malignant disease progression & $62(32.6)$ \\
\hline $\begin{array}{l}\text { median time to malignant disease progres- } \\
\text { sion }\end{array}$ & 3.96 yrs (4-148 mos) \\
\hline \multicolumn{2}{|c|}{$\begin{array}{l}\text { * EDH = epidural hematoma; LL = lower limb; SMA = supplementary } \\
\text { motor area; UL = upper limb. } \\
\dagger \text { The patient developed a new mild hemiparesis that completely re- } \\
\text { solved after evacuation. } \\
\ddagger \text { Determined on the basis of pre- and postoperative T2-weighted MRI } \\
\text { studies, following the methodological procedure described by Smith et } \\
\text { al. }{ }^{38} \\
\S \text { Since the first operation. }\end{array}$} \\
\hline
\end{tabular}

For this purpose, the study population was divided into the following 2 subgroups: Subgroup A, patients with $\Delta$ VT2T1 values smaller than $30 \mathrm{~cm}^{3}$ (116 patients); and Subgroup B, patients with a $\Delta$ VT2T1 value of $30 \mathrm{~cm}^{3}$ or larger (74 patients).

Our results showed that Subgroup A had a median EOR of $91 \%$, while Subgroup B had a median EOR of $79 \%$, $(\mathrm{z}=4.75$, Mann-Whitney test; $\mathrm{p}<0.0001$ ) (Table $2)$. Even considering EOR as an ordinal variable $(<70 \%$, $70 \%-89 \%$, and $\geq 90 \%$ ), the association with the $\Delta \mathrm{VT} 2 \mathrm{~T} 1$ value subgroups remains significant (Table 3 ). In particular, the association between postoperative tumor volume on T2-weighted MRI images and $\Delta \mathrm{VT} 2 \mathrm{~T} 1$ value $\left(\mathrm{r}_{\mathrm{s}}=\right.$ $0.77, p<0.0001)$ is illustrated in Fig. 2, taking into consideration the percentage of the EOR volume also.

Consequently, our data show that the greater the preoperative $\Delta \mathrm{VT} 2 \mathrm{~T} 1$ value, the less extensive the resection currently possible; this finding points to a possible predictive correlation between $\Delta \mathrm{VT} 2 \mathrm{~T} 1$ value and EOR achievable.

\section{Overall Survival}

Overall, 41 patients died (21.6\%), and the median follow-up in the surviving patients was 4.7 years (range 4-155 months). The estimated 5- and 8-year OS rates were $80 \%$ and $66 \%$, respectively. The prognostic factors, associated ( $\mathrm{p} \leq 0.15)$ with OS in the univariate proportional hazard model, were as follows: age, log preopera- 


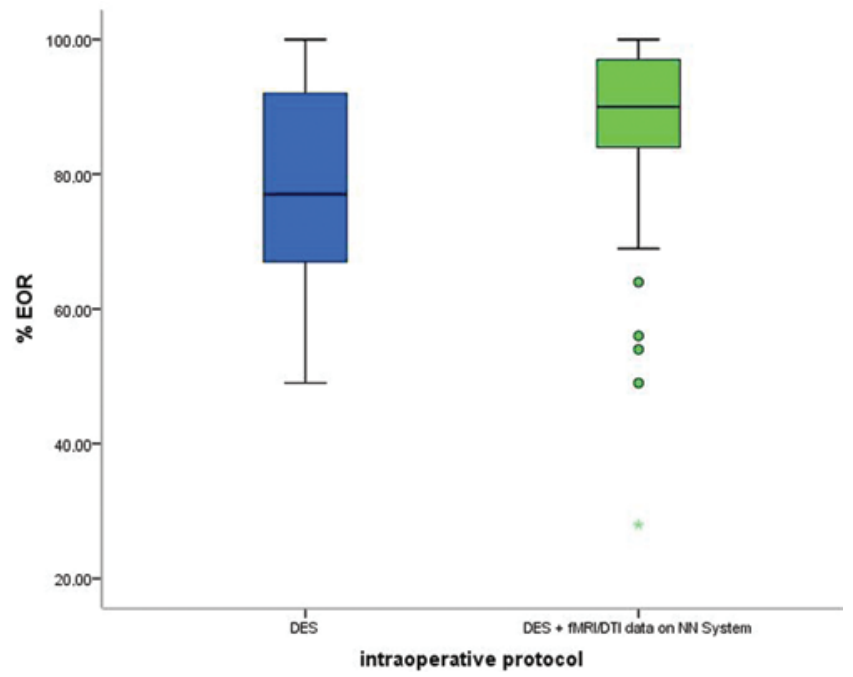

FIG. 1. Box plot showing the difference achieved in tumor resection between Series 1 (blue box) and Series 2 (green box). Series 1 had a mean EOR of $77 \%$, while Series 2 had a mean EOR of $90 \%$. The intraoperative use of a guided navigation system (NN) enriched with $\mathrm{fMRI} / \mathrm{DTI}$ data, in addition to IES used in the second series, significantly increased the EOR achieved. The asterisk represents the outlier, and the circles indicate the suspected outliers. DES = direct electrical stimulation.

tive tumor volume, intraoperative protocol used, histological tumor subtype, EOR, $\Delta \mathrm{VT} 2 \mathrm{~T} 1$ value, and postoperative residual tumor.

Univariate analysis results are summarized in Table 4. An increase in age and in log preoperative tumor volume were associated with a significantly worse OS, while the introduction of fMRI/DTI data on the neuronavigation system (Series 2 vs Series 1) improved the EOR and consequently the OS.

The OS was also significantly improved (HR 4.262 [95\% CI 1.748-10.388], $\mathrm{p}=0.001$ ) for patients with histological diagnosis of oligodendrogliomas compared to those with fibrillary astrocytomas. The estimated 5- and 8 -year OSs were $88 \%$ and $85 \%$ for patients with WHO Grade II oligodendrogliomas and $73 \%$ and $44 \%$ for patients with astrocytomas, respectively.

No significant differences were found between patients with a histological diagnosis of oligoastrocytoma compared with those with oligodendroglioma $(p=0.636)$ (Fig. 3A). Furthermore, the EOR, treated as a continuous variable, was associated with a significant improvement in OS (HR 0.93 [95\% CI 0.92-0.95], p < 0.0001).

With EOR divided into 3 categories, the estimated 5-

TABLE 3: Linear association between $\triangle \mathrm{VT} 2 \mathrm{~T} 1$ and EOR*

\begin{tabular}{lccc}
\hline & \multicolumn{3}{c}{ No. of Patients (\%) } \\
\cline { 2 - 4 }$\%$ EOR & $\Delta \mathrm{VT} 2 \mathrm{~T} 1<30 \mathrm{~cm}^{3}$ & $\Delta \mathrm{VT} 2 \mathrm{~T} 1 \geq 30 \mathrm{~cm}^{3}$ & Total \\
\hline$\geq 90$ & $68(58.62)$ & $22(29.73)$ & $90(47.4)$ \\
$70-89$ & $42(36.21)$ & $28(37.84)$ & $70(36.8)$ \\
$<70$ & $6(5.17)$ & $24(32.43)$ & $30(15.8)$ \\
total & 116 & 74 & 190 \\
\hline
\end{tabular}

* Pearson $\chi^{2}=29.256$, df $2, p<0.001$.

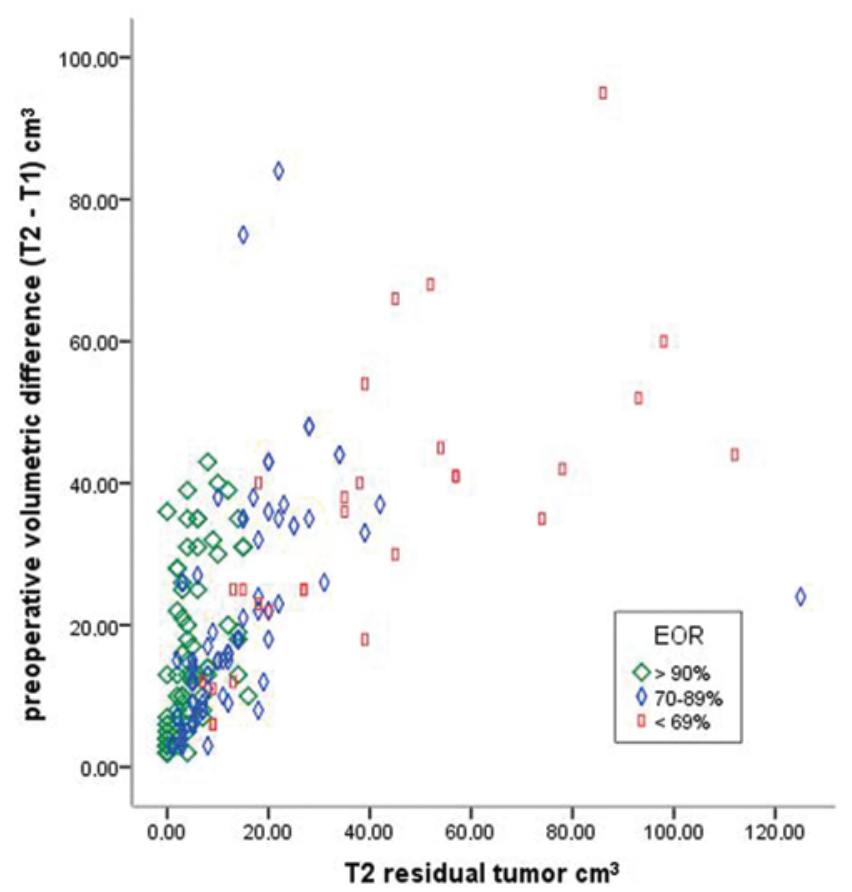

Fıg. 2. Graph showing the association between postoperative tumor volumes computed on T2-weighted MRI studies and preoperative $\Delta$ VT2T1 values $\left(r_{s}=0.77, p<0.0001\right)$. Although there is a tendency toward higher EOR $(\geq 90 \%)$ and smaller tumors, the data highlight a correlation tendency between a higher $\triangle \mathrm{VT} 2 \mathrm{~T} 1$ value and a lesser EOR achieved. A major volumetric difference between T2- and contrast-enhanced T1-weighted MRI sequences suggests a greater propensity of the tumor to have a diffuse growing pattern and consequently to be less resectable.

and 8 -year OS rates were $93 \%$ and $89 \%$ for patients with an EOR of $90 \%$ or more; $84 \%$ and $59 \%$ for patients with an EOR of $70 \%-89 \%$; and $41 \%$ and $17 \%$ for patients with an EOR less than 70\%, respectively (Fig. 3B).

Overall, our results show that patients with an EOR less than 70\% had a risk of death 19.7 (95\% CI 7.08-54.8) times higher than patients with an EOR of $90 \%$ or more.

The impact of residual tumor volume on OS was also analyzed. Patients with a smaller postoperative tumor volume had a significantly better OS. Considering the 4 subgroups defined according to the residual tumor volume value $\left(<10 \mathrm{~cm}^{3}, 10-19 \mathrm{~cm}^{3}, 20-29 \mathrm{~cm}^{3}\right.$, and $\leq$ $30 \mathrm{~cm}^{3}$ ), the estimated 5-year OS rates were $93 \%, 85 \%$, $57 \%$, and $37 \%$, respectively, and the estimated 8-year OS rates were $87 \%, 56 \%, 57 \%$, and $14 \%$, respectively (Fig. $3 \mathrm{C})$. Moreover, the univariate Cox proportional hazard analysis showed that the $\Delta \mathrm{VT} 2 \mathrm{~T} 1$ value, treated as a continuous variable, was associated with a worse OS (HR 1.04 [95\% CI 1.03-1.06], $\mathrm{p}<0.0001)$.

For graphic visualization purposes, the $\Delta \mathrm{VT} 2 \mathrm{~T} 1$ value was divided into 2 categories as follows: $\Delta$ VT2T1 value less than $30 \mathrm{~cm}^{3}$ and $\Delta V T 2 T 1$ value $30 \mathrm{~cm}^{3}$ or higher. The estimated 5- and 8-year OS rates were $91 \%$ and $78 \%$ for a $\Delta \mathrm{VT} 2 \mathrm{~T} 1$ value less than $30 \mathrm{~cm}^{3}$ and $56 \%$ and $38 \%$ for a $\Delta \mathrm{VT} 2 \mathrm{~T} 1$ value of $30 \mathrm{~cm}^{3}$ or higher, respectively (Fig. 3D).

In particular, this analysis shows that patients with a $\Delta \mathrm{VT} 2 \mathrm{~T} 1$ value of $30 \mathrm{~cm}^{3}$ or higher were at a risk of death 
Low-grade glioma surgery in eloquent areas

TABLE 4: Univariate analysis of clinical data, tumor parameters, and volumetric tumor data with OS, PFS, and MPFS in 190 patients with LGGs*

\begin{tabular}{|c|c|c|c|c|c|c|c|c|c|}
\hline \multirow[b]{2}{*}{ Factor } & \multicolumn{3}{|c|}{ OS } & \multicolumn{3}{|c|}{ PFS } & \multicolumn{3}{|c|}{ MPFS } \\
\hline & $\mathrm{HR}$ & $95 \% \mathrm{Cl}$ & p Value & $\mathrm{HR}$ & $95 \% \mathrm{Cl}$ & p Value & $\mathrm{HR}$ & $95 \% \mathrm{Cl}$ & $p$ Value \\
\hline age† & 1.030 & $1.007-1.055$ & 0.011 & 1.006 & $0.988-1.025$ & 0.513 & 1.015 & $0.995-1.036$ & 0.137 \\
\hline male & 1 & & & 1 & & & 1 & & \\
\hline female & 0.709 & $0.366-1.372$ & 0.308 & 1.324 & $0.848-2.066$ & 0.220 & 0.780 & $0.460-1.323$ & 0.352 \\
\hline \multicolumn{10}{|l|}{ tumor location } \\
\hline precentral area & 1 & & & 1 & & & 1 & & \\
\hline insular lobe & 1.642 & $0.189-3.291$ & 0.162 & 1.691 & $1.022-2.797$ & 1.141 & 1.358 & $0.786-2.345$ & 0.272 \\
\hline temporal lobe & 1.164 & $0.450-3.011$ & 0.753 & 1.044 & $0.584-2.145$ & 0.906 & 0.868 & $0.392-1.921$ & 0.729 \\
\hline postcentral area & 1.269 & $0.363-4.433$ & 0.708 & 2.107 & $0.948-4.682$ & 0.067 & 0.533 & $0.125-2.257$ & 0.393 \\
\hline \multicolumn{10}{|l|}{ tumor subtype } \\
\hline oligodendroglioma & 1 & & & 1 & & & 1 & & \\
\hline oligoastrocytoma & 1.332 & $0.405-4.373$ & 0.636 & 1.078 & $0.533-2.181$ & 0.833 & 1.078 & $0.533-2.181$ & 0.833 \\
\hline fibrillary astrocytoma & 4.262 & $1.748-10.388$ & 0.001 & 2.273 & $1.326-3.895$ & 0.003 & 2.273 & $1.326-3.895$ & 0.003 \\
\hline \multicolumn{10}{|l|}{ intraop protocol } \\
\hline Series 1 & 1 & & & 1 & & 1 & & & \\
\hline Series 2 & 0.388 & $0.179-0.839$ & 0.016 & 0.969 & $0.604-1.553$ & 0.056 & 0.600 & $0.336-1.072$ & 0.085 \\
\hline$\%$ EOR† & 0.933 & $0.915-0.952$ & $<0.0001$ & 0.930 & $0.915-0.946$ & $<0.0001$ & 0.944 & $0.928-0.960$ & $<0.0001$ \\
\hline \multicolumn{10}{|l|}{$\%$ EOR } \\
\hline$\geq 90$ & 1 & & & 1 & & & 1 & & \\
\hline $\begin{array}{l}\text { postop tumor vol on T2- } \\
\text { weighted images } †\end{array}$ & 1.022 & $1.014-1.031$ & $<0.0001$ & 1.023 & $1.016-1.029$ & $<0.0001$ & 1.021 & $1.013-1.029$ & $<0.0001$ \\
\hline \multicolumn{10}{|l|}{$\begin{array}{l}\text { postop tumor vol on T2- } \\
\text { weighted images }\end{array}$} \\
\hline$<10 \mathrm{~cm}^{3}$ & 1 & & & 1 & & & 1 & & \\
\hline $10-20 \mathrm{~cm}^{3}$ & 3.281 & $1.340-8.035$ & 0.009 & 1.746 & $0.946-3.222$ & 0.074 & 1.536 & $0.795-2.967$ & 0.200 \\
\hline $21-30 \mathrm{~cm}^{3}$ & 6.500 & $2.434-17.353$ & $<0.0001$ & 5.842 & 3.049-11.191 & $<0.0001$ & 2.982 & $1.368-6.501$ & 0.006 \\
\hline$>31 \mathrm{~cm}^{3}$ & 13.980 & $5.677-34.423$ & $<0.0001$ & 13.061 & $6.961-24.506$ & $<0.0001$ & 8.739 & $4.407-17.329$ & $<0.0001$ \\
\hline \multicolumn{10}{|l|}{ postop chemotherapy } \\
\hline no & 1 & & & 1 & & & 1 & & \\
\hline yes & 1.395 & $0.584-3.330$ & 0.436 & 1.005 & $0.572-1.767$ & 0.986 & 1.178 & $0.595-2.332$ & 0.639 \\
\hline \multicolumn{10}{|l|}{ postop EBRT } \\
\hline no & 1 & & & 1 & & & 1 & & \\
\hline yes & 0.598 & $0.323-1.106$ & 0.101 & 0.600 & $0.385-0.935$ & 0.024 & 0.578 & $0.351-0.954$ & 0.032 \\
\hline
\end{tabular}

* Values in boldface are statistically significant from 2-sided tests (Cox regression) and were statistically significant when $p<0.05$.

$\uparrow$ Modeled as a continuous variable. 
T. Ius et al.
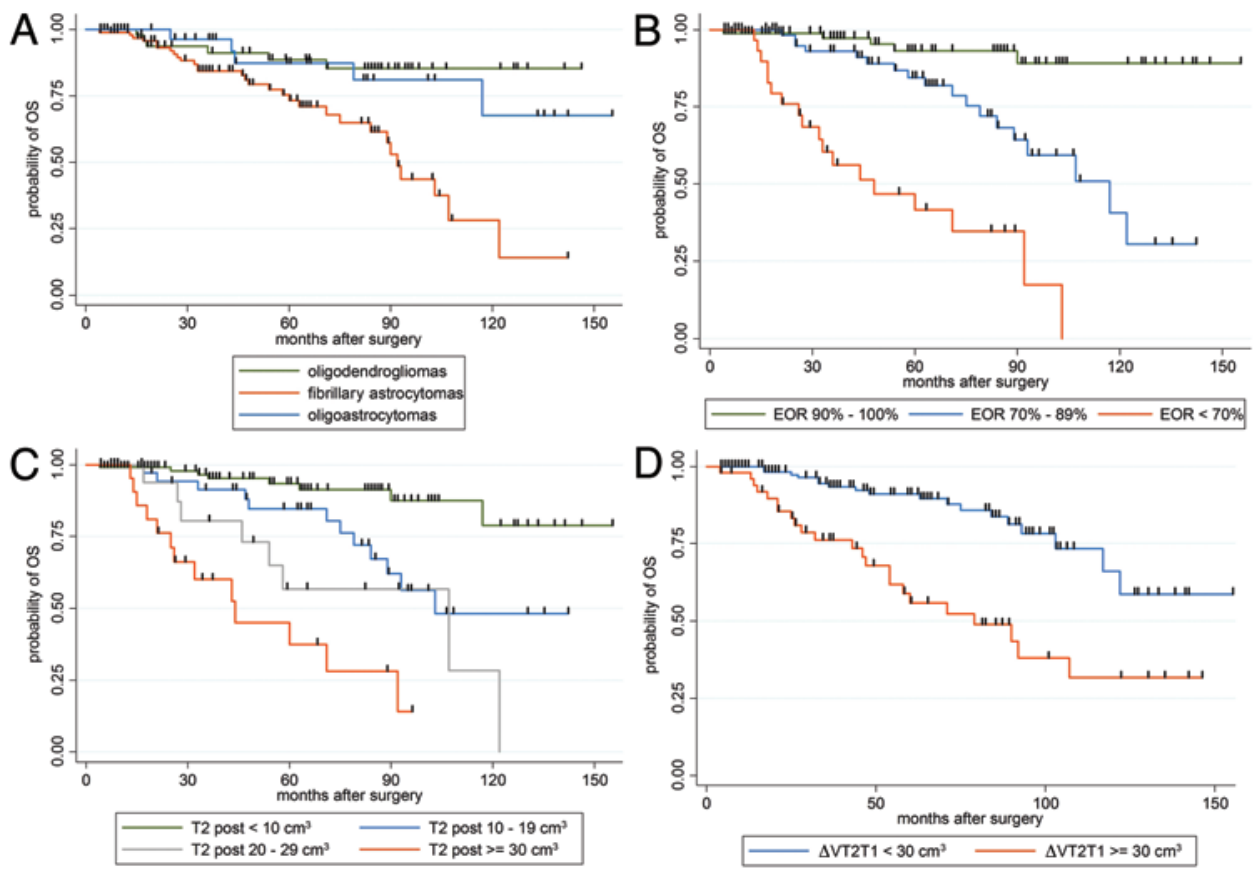

FIG. 3. Kaplan-Meier curves revealing the OS in patients with LGGs, stratified by histological subtype (A), EOR (B), postoperative residual tumor volume computed on T2-weighted images (T2 post) (C), and preoperative $\Delta V T 2 T 1$ value (D). Patients with a diagnosis of oligodendroglioma, EOR of at least $90 \%$, residual tumor smaller than $10 \mathrm{~cm}^{3}$, and $\Delta V T 2 T 1$ value less than $30 \mathrm{~cm}^{3}$ have significantly longer OS.

4 times greater than patients with a preoperative difference of less than $30 \mathrm{~cm}^{3}$ (HR 3.70 [95\% CI 1.99-6.87], p $<0.0001$ ) due to the greater difficulty of complete resection of the glioma without excessive postoperative morbidity.

Finally, multivariate Cox analysis showed that OS was significantly worse in older patients, as well as in patients with a histological diagnosis of fibrillary astrocytoma, as compared to those with oligodendroglioma and to patients with higher $\Delta \mathrm{VT} 2 \mathrm{~T} 1$ values (Table 5).

\section{Tumor Progression and Malignant Transformation}

Tumor progression was identified in 79 patients (41.6\%) and malignant progression was noted in 62 patients $(32.6 \%)$, as indicated by new contrast enhancement on follow-up imaging (Table 2). Forty-eight of the 62 patients with malignant progression underwent a second surgery, and a higher-grade tumor was identified. Among the remaining 14 patients with MPFS in whom a second surgery was not performed, 9 received EBRT before malignant progression.

The median PFS and MPFS were 3.5 years (range 4-155 months) and 3.96 years (range 4-148 months), respectively. The estimated 5- and 8-year survival rates were $59 \%$ and $35 \%$ for PFS and $74 \%$ and $47 \%$ for MPFS, respectively.

Univariate analysis showed that the following prognostic factors were associated with PFS and MPSF ( $p$ $<0.05$ ): $\log$ preoperative tumor volume, tumor subtype, EOR, $\triangle \mathrm{VT} 2 \mathrm{~T} 1$ value, residual tumor, and EBRT. These results are summarized in Table 4. In particular, a higher log preoperative tumor volume was significantly associated with a worsening of both PFS and MPFS. As far as histological subtype data are concerned, PFS was significantly improved for patients with histological diagnosis of oligodendroglioma compared with those with fibrillary

TABLE 5: Variables independently associated with OS, PFS, and MPFS after resection of LGGs in a multivariate proportional hazard analysis (Cox model)*

\begin{tabular}{|c|c|c|c|c|c|c|c|c|c|}
\hline \multirow[b]{2}{*}{ Factor } & \multicolumn{3}{|c|}{ OS } & \multicolumn{3}{|c|}{ PFS } & \multicolumn{3}{|c|}{ MPFS } \\
\hline & HR & $95 \% \mathrm{Cl}$ & $p$ Value & $\mathrm{HR}$ & $95 \% \mathrm{Cl}$ & $p$ Value & $\mathrm{HR}$ & $95 \% \mathrm{Cl}$ & $p$ Value \\
\hline age† & 1.035 & $1.011-1.060$ & 0.003 & & NS & & & NS & \\
\hline histological subtypeł & 2.974 & $1.401-6.315$ & 0.005 & & NS & & 3.202 & $1.489-6.886$ & 0.003 \\
\hline$\%$ EOR† & 0.958 & $0.936-0.981$ & 0.001 & 0.940 & $0.924-0.956$ & $<0.0001$ & 0.963 & $0.944-0.982$ & $<0.0001$ \\
\hline$\Delta \mathrm{VT} 2 \mathrm{~T} 1 \dagger$ & 1.035 & $1.017-1.054$ & $<0.0001$ & 1.021 & $1.008-1.034$ & 0.001 & 1.029 & $1.014-1.045$ & $<0.0001$ \\
\hline
\end{tabular}

* NS = not significant in multivariate Cox analysis.

$\dagger$ Modeled as a continuous variable.

‡ Fibrillary astrocytoma versus oligodendroglioma and oligoastrocytoma. 
astrocytoma, while both oligoastrocytoma and fibrillary astrocytoma were associated with a worse MPFS than oligodendroglioma.

The EOR, treated as both a continuous and an ordinal variable, was associated with significant improvement of both PFS and MPFS values. The Kaplan-Meier curves demonstrating the PFS and MPFS in patients with LGGs, stratified by EOR subgroups, are shown in Figs. 4A and $5 \mathrm{~A}$, respectively. In quantitative terms, our analysis shows that patients with an EOR of less than $70 \%$ have a much higher risk of disease progression and malignant transformation than do patients with an EOR of $90 \%$ or more (13.6 [95\% CI 7.18-25.79] times higher risk for disease progression and 9.77 [95\% CI 4.85-19.65] times higher for malignant progression).

Similarly, patients with a smaller postoperative tumor volume showed significantly longer periods of PFS and MPFS. The PFS and MPFS estimates for 4 residual tumor volume subgroups are shown in Figs. $4 \mathrm{~B}$ and 5B, respectively.

An increase in $\triangle \mathrm{VT} 2 \mathrm{~T} 1$ value, modeled as both continuous and dichotomous variables, was associated with an earlier tumor progression as well as malignant transformation. The Kaplan-Meier PFS and MPFS plots, estimated for $\triangle \mathrm{VT} 2 \mathrm{~T} 1$ as a dichotomous variable, are shown in Figs. $4 \mathrm{C}$ and $5 \mathrm{C}$, respectively.

Finally, multivariate Cox analysis shows that PFS was significantly worse in patients with higher $\Delta \mathrm{VT} 2 \mathrm{~T} 1$ values and histological diagnosis of fibrillary astrocytoma versus oligodendroglioma. Conversely, higher EOR values and lower $\Delta$ VT2T1 values were associated with a longer PFS.

In the final model, MPFS was shown to be significantly worse in patients with higher $\Delta \mathrm{VT} 2 \mathrm{~T} 1$ values, histological diagnosis of oligoastrocytoma, and fibrillary astrocytomas versus oligodendrogliomas, whereas higher EOR values and lower $\triangle \mathrm{VT} 2 \mathrm{~T} 1$ values were associated with a better MPFS (Table 5).

\section{Discussion}

Low-grade gliomas are infiltrative cerebral tumors, characterized by a systematic progression to malignant transformation. ${ }^{19,50,55,59}$ In the literature, 5-year survival ranges from $42 \%$ to $98 \%$. $16,31,32,35,38,42,43,58,61$ To date, there is still no general consensus regarding the effect of EOR on OS among patients with LGGs, due to the lack of Class I evidence. However, since 1990, many different studies have been published evaluating the benefit of the maximum EOR of glioma in improving OS and delaying tumor progression. $1,15,27,32,35,38,39,42,43,49,52,54,58,60,61$ The most critical point in achieving a radical resection in LGGs is represented by their tendency to infiltrate eloquent cortical areas and subcortical pathways. ${ }^{20,34}$

\section{Extent of Resection and Surgical Technique}

Recent advances in microsurgical and brain mapping techniques with neurophysiological monitoring have led to an increase in the use of surgery for LGGs. The neuroradiological techniques, such as fMRI and 3D reconstruction of white matter tracts, represent useful tools to preoperatively analyze the tridimensional relationships between the tumor and the neighboring cortical functional areas and subcortical pathways, respectively. 2,9,29,52,56

The fMRI/DTI data loaded into the neuronavigation system, which are available intraoperatively, may provide useful intraoperative guidance. , $, 8,52^{3}$ It was demonstrated that the location and course of the tract were dependent on the characteristics of the tumor, such as histology, edema, location,, 310 and on some technical factors, such as the fractional anisotropy threshold used for start and stop tracking. ${ }^{4,30,37}$ Consequently, all functional areas and subcortical pathways must be continuously verified with IES. In addition to IES, we have recently introduced enlarged, continuous, and variant neuropsychological testing, which is helpful during subcortical stimulation on the dominant side. Our results demonstrate that the intraoperative use of a guided navigation system enriched with functional and DTI data used in Series 2 significantly increase the EOR possible. Patients in Series 1 had a mean EOR of $77 \%$, while those in Series 2 had a mean EOR of 90\% ( $\mathrm{p}=0.0001)$.

Specifically, the combination of DTI fiber tracking and IES allowed the accurate identification of eloquent fiber tracts and increased surgical performance, maintaining a high rate of functional preservation. In addition, the routine use of intraoperative transcranial motor evoked potential monitoring is helpful in preventing direct injury to the pyramidal tract. ${ }^{51,52}$ In our experience, motor evoked potentials are useful in warning us about direct
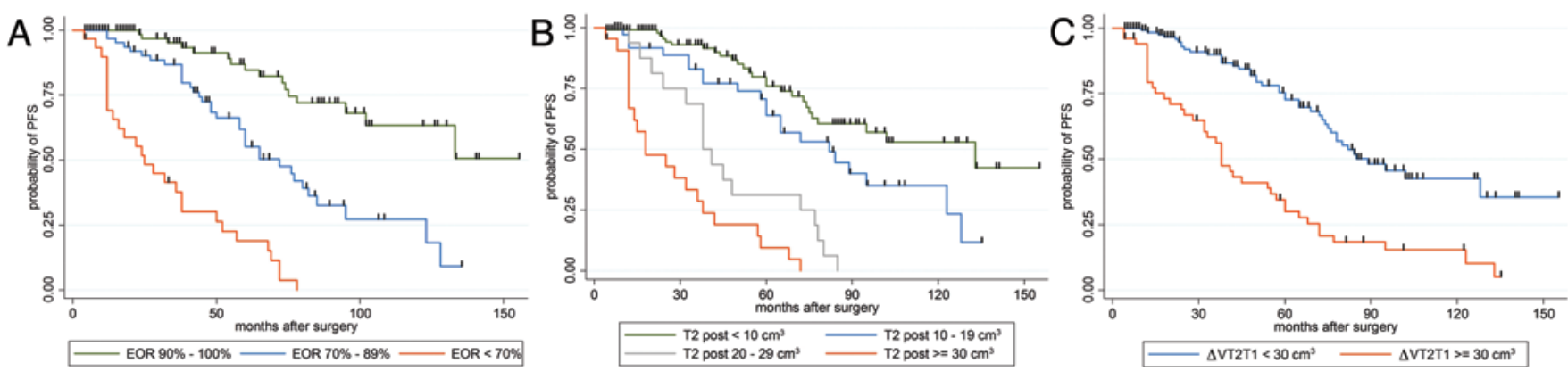

FIG. 4. Kaplan-Meier curves revealing the PFS in patients with LGGs, stratified by EOR (A), postoperative residual tumor volume (T2 post) (B), and $\Delta$ VT2T1 value (C). Patients with EOR of at least $90 \%$, preoperative $\Delta V T 2 T 1$ smaller than $30 \mathrm{~cm}^{3}$, and postoperative residual tumor volume computed on T2-weighted images smaller than $10 \mathrm{~cm}^{3}$ have a significant delay in tumor progression. 
T. Ius et al.
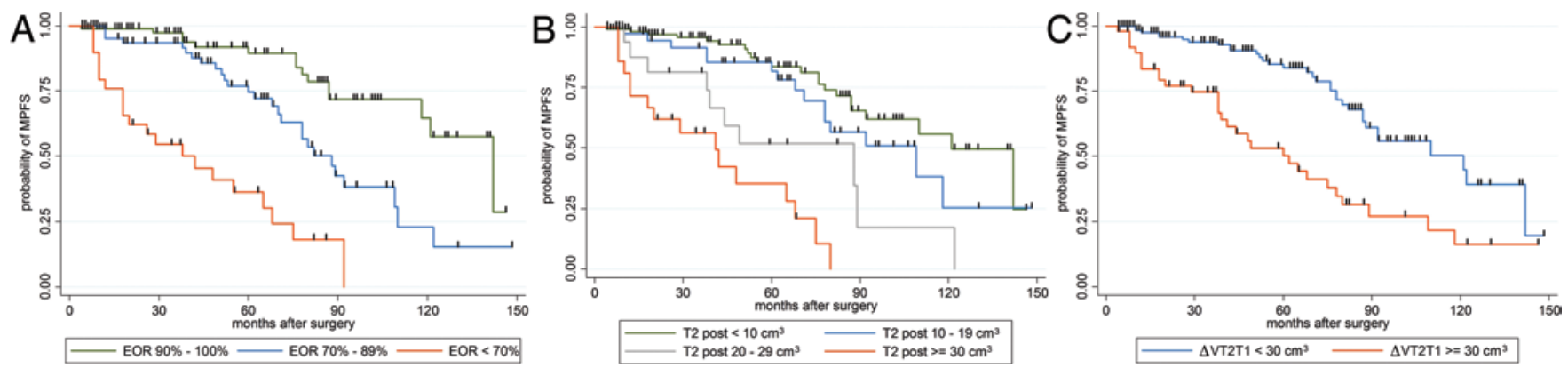

FIG. 5. Kaplan-Meier curves revealing the MPFS in patients with LGGs, stratified by EOR (A), postoperative residual tumor volume computed on T2-weighted images (T2 post) (B), and preoperative $\triangle \mathrm{VT} 2 \mathrm{~T} 1$ (C). Patients with EOR equal to or greater than $90 \%$, with $\Delta \mathrm{VT} 2 \mathrm{~T} 1$ less than $30 \mathrm{~cm}^{3}$, and with postoperative residual tumor volume computed on T2-weighted images less than $10 \mathrm{~cm}^{3}$ demonstrate an improvement in MPFS.

mechanical trauma to the fibers, but they do not guarantee the prevention of vascular damage.

Finally, we always use intraoperative electrocorticography, which is, in our opinion, essential to record afterdischarge phenomena and electrical and clinical seizures. In particular, short-duration focal seizures, which can interfere with the patient's collaboration during mapping, may be highlighted.

\section{Volumetric and Survival Analysis}

Until recently, EOR has often been estimated solely on the basis of the intraoperative interpretation of the surgeon, resulting in erroneous conclusions about the actual role of surgery. ${ }^{19}$ No studies have randomized the patients with respect to EOR, and in only 5 recent studies was the volumetric analysis of EOR applied. . $^{16,49,54,55,58}$

Among the nonvolumetric studies, 8 demonstrated evidence supporting EOR as a statistically significant predictor of 5-year survival by comparing gross-total resection, near-total resection, and subtotal resection, $1,15,32,35,38,39,42,61$ while all volumetric studies showed evidence supporting EOR as a statistically significant predictor of OS by a combination of multivariate and univariate analyses (Table 6).

All volumetric investigations demonstrated that a more aggressive resection predicts a significant improvement in OS, ${ }_{16,49,52,52,58}$ although the cutoff threshold of EOR is different in each publication and varied from $75 \%$ to $100 \%$. Van Veelen et al. ${ }^{58}$ and Claus et al. ${ }^{16}$ were the first to assess the volumetric evaluation of EOR in LGG surgery, reporting 5-year Kaplan-Meier estimations of survival of $62 \%$ and $92 \%$, respectively, for tumor resection greater than $75 \%$.

Most recently, Smith et al. ${ }^{54}$ performed a retrospective volumetric analysis of EOR in 216 patients with LGGs and found that patients with at least $90 \%$ resection had 5- and 8-year OSs of 97\% and 91\%, respectively, whereas patients with less than $90 \%$ resection had 5- and 8 -year OS rates of $76 \%$ and $60 \%$, respectively. In line with these papers, our volumetric analysis provides further evidence that a more aggressive resection correlates with a significant improvement in OS ( $\mathrm{p}=0.001)$, as well as in PFS ( $p<0.0001)$ and MPFS $(\mathrm{p}<0.0001)$, compared with a simple debulking procedure.

Overall, the present volumetric analysis shows 5-year
OS rates, 5-year PFS, and 5-year MPFS rates of 93\%, $88 \%$, and $91 \%$ respectively, when EOR was at least $90 \%$. In addition, the multivariate analysis in our study population shows that EOR is an independent prognostic factor for OS, as well as for PFS and MPFS.

Since oligodendrogliomas, particularly those with $1 \mathrm{p} 19 \mathrm{q} \mathrm{LOH}$, have more benign courses, ${ }^{25,26}$ we separated these histological subtypes as a covariate in a multivariate analysis. Our analysis, as previously reported by McGirt et al., ${ }^{35}$ showed that patients with oligodendroglioma and oligoastrocytoma have a better prognosis with respect to those with fibrillary astrocytoma (5-year OS rates of $88 \%$ vs 5-year OS rates of $87 \%$ and $73 \%$, respectively), confirming a different natural history between low-grade oligodendroglioma-oligoastrocytoma and low-grade astrocytomas. ${ }^{50}$ In our series, $1 \mathrm{p} 19 \mathrm{q}$ status was assessed in the minority of specimens; therefore, survival comparison between LOH-negative and LOH-positive LGGs was not possible because only 14 cases of oligodendrogliomas were proven to have $1 \mathrm{p} 19 \mathrm{q} \mathrm{LOH}$.

Finally, in our investigation of the impact of residual tumor volume on OS, PFS, and MPFS, we found a significant advantage for all outcome measures when the residual volume was smaller than $10 \mathrm{~cm}^{3}$. Indeed, the decrease in the number of remaining cells theoretically decreases the risk of malignant cellular degeneration and hence potentially prolongs OS..$^{25,54}$

As far as the methodological procedure is concerned, the present study was designed to follow the methodology proposed by Smith et al. ${ }^{54}$ for the assessment of EOR along with with careful consideration of the limitations of many previous studies.

We recruited only adult patients and excluded cases of pilocytic astrocytoma (WHO Grade I), gemistocytic astrocytoma, and patients treated with upfront radiation therapy or chemotherapy to create a more uniform study population. Nonetheless, our study also has limitations. First, it is a retrospective study, and this analytical approach does not allow for a standardized patient followup. Moreover, patients who underwent a biopsy procedure were excluded; therefore, our data cannot offer a direct comparison between patients undergoing biopsy only and patients undergoing maximum resection. Furthermore, the end points of PFS and MPFS have several potential limitations. New contrast enhancement can appear after 
TABLE 6: Statistical relationship of EOR to OS and PFS in patients with LGGs in a review of the literature*

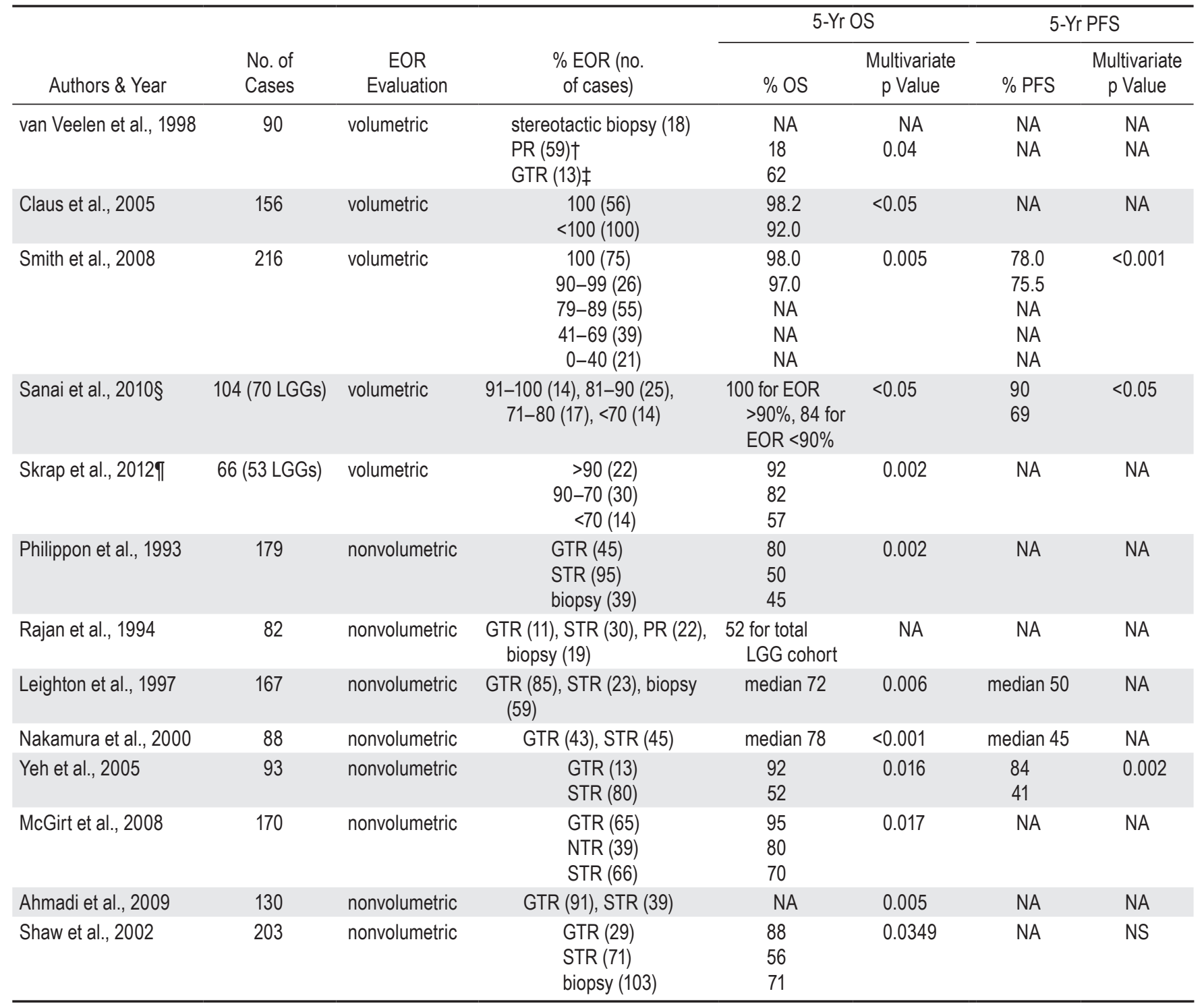

\footnotetext{
* Since 1990, many different nonvolumetric and volumetric studies have used statistical analysis to examine the role of EOR in improving survival and delaying tumor progression in patients with LGGs, demonstrating a survival benefit for greater EOR. The overall trend favors the value of greater EOR in improving patient outcome. Abbreviations: GTR = gross-total resection; NA = not assessed; NS = not significant; NTR = near-total resection; PR = partial resection; STR = subtotal resection.

$\dagger$ Estimated tumor removal $<75 \%$.

$\ddagger$ Estimated tumor removal $>75 \%$.

$\S$ Paper focused on insular glioma surgery.

II Paper focused on insular nonenhancing glioma surgery.
}

radiotherapy; ${ }^{53}$ in the present series, in 14 of 62 patients with MPFS ( 9 of whom received EBRT before malignant progression), the diagnosis of malignant transformation was made on the basis of imaging alone without histological confirmation. We recognize that it could be difficult to distinguish radiation effects from progression; however, these 9 patients diagnosed with malignant progression on the basis of imaging alone were far enough out from EBRT to expect that any effect of treatment on imaging results had been resolved. A further limita- tion is that at the time of tumor progression or malignant transformation, patients were treated with nonstandardized chemo- and radiotherapeutic protocols. Lastly, the molecular markers, which are increasingly used for the assessment and management of LGGs, were not included in the multivariate analysis due to their recent discovery and introduction in clinical practice. ${ }^{12,13,55}$

\section{Clinical Role of $\triangle V T 2 T 1$ Value and Future Perspectives}

The major limitation in achieving a complete resec- 
tion is the infiltrative growing pattern of these lesions, especially at the subcortical level. Consequently, the definition of the best management of a residual tumor represents the main open question in clinical practice. ${ }^{12,55}$ To this end, neoadjuvant chemotherapy was recently advocated for LGG management, with the aim of inducing tumor shrinkage before operation or reoperation. ${ }^{12,19,55}$ As it is usually believed that postoperative tumor volume influences the prognosis for patients with this disease, $,, 17,28,61$ it may be more advantageous to start active treatment with radiotherapy or even chemotherapy. ${ }^{12-14}$ It is therefore of the utmost importance to develop a standard tool that enables an objective preoperative estimation of the postoperative tumor volume. Lang et al ${ }^{30}$ first asserted that lesions with diffuse margins on T2-weighted MRI are less amenable to radical resection than those with sharp margins. Recently, Skrap et al. ${ }^{52}$ introduced a preoperative method to discriminate the proliferative growing mechanism from the diffusive spreading mechanism along the white matter and to assess preoperatively the degree of resection. Data reported showed that when proliferation is the major diffusivity phenomenon, it does not affect the tumor shape (resulting in a regular shape, comparable on both preoperative contrast-enhanced T1- and T2-weighted MRI studies), which is grossly bulky. By contrast, when the diffusive pattern is predominant, the tumor will result in a complex shape with digitations along the white matter, which is more visible on T2-weighted images. ${ }^{34,52}$

In the present study, our findings confirmed the data reported by Skrap et al. ${ }^{52}$ on the role of the $\Delta \mathrm{VT} 2 \mathrm{~T} 1$ value in OS and in representing a prognostic preoperative index of EOR itself. Our findings also confirmed the role of this preoperative value in tumor progression and malignant transformation. Patients with a $\Delta$ VT2T1 value of less than $30 \mathrm{~cm}^{3}$ have a better prognosis than those with a $\Delta \mathrm{VT} 2 \mathrm{~T} 1$ value more than $30 \mathrm{~cm}^{3}$ in terms of OS ( $\left.<<0.0001\right)$, PFS ( $\mathrm{p}<0.0001)$, and MPFS ( $<<0.0001)$. Even after having applied a multivariate analysis, the $\Delta \mathrm{VT} 2 \mathrm{~T} 1$ value was found to be a significant preoperative prognostic factor for OS ( $p<0.0001)$, PFS ( $\mathrm{p}=0.001)$, and MPFS ( $\mathrm{p}<$ 0.0001 ). Moreover, our results show that in cases with a $\Delta$ VT2T1 value less than $30 \mathrm{~cm}^{3}$ (Subgroup A) the median EOR was $91 \%$, whereas when this difference was more than $30 \mathrm{~cm}^{3}$ (Subgroup B) the mean EOR was 79\% (p < 0.0001). Thus, the present investigation confirms the possibility of identifying in the $\Delta$ VT2T1 value a prognostic preoperative index of EOR itself. The significant correlation between the preoperative $\triangle \mathrm{VT} 2 \mathrm{~T} 1$ value and the EOR indicates that this index is a promising tool to use in the decision-making process over whether to operate on a LGG or start preoperative neoadjuvant chemotherapy to reduce the infiltrative growing pattern of the tumor and, consequently, to increase the possibility of a total resection. Finally, for patients who undergo chemotherapy as an initial treatment or at recurrence after a previous partial resection due to the invasion of functional subcortical pathways, the $\triangle \mathrm{VT} 2 \mathrm{~T} 1$ value may represent a useful tool to assess the efficacy of the treatment itself and its effectiveness in decreasing the infiltrative tumor component.

\section{Conclusions}

In line with recent volumetric studies on the objective evaluation of EOR, our data confirm the role of surgery as the first-line treatment for LGGs. The EOR is a strong independent factor associated with improved OS, as well as with delay of progression and malignant transformation. Due to intraoperative corticosubcortical mapping, neurophysiological monitoring, and overlap of fMRI/DTI data on the neuronavigation system, a major resection is possible with an acceptable risk and a significant improvement in long-term patient outcomes. However, the major limitation in achieving a complete resection is due to the infiltrative growing pattern of these lesions, particularly at the subcortical level. Consequently, the preoperative assessment of the prevailing pattern of growth (infiltrative vs proliferative), by computing the $\Delta \mathrm{VT} 2 \mathrm{~T} 1$ value, may constitute a predictive index of EOR itself. This index could also represent a new tool to help with surgical decision making and therefore standardize the therapeutic strategy in LGG management. In addition, how to best manage the residual tumor represents the main open question in clinical practice, and the impact of the residual tumor on OS, PFS, and MPFS should also be investigated in the context of molecular prognostic factors to define better postoperative standardized adjuvant protocols. Finally, to develop a dynamic strategy for the management of LGGs, further prospective studies focusing on detailed molecular and genetic differentiation among LGGs should be undertaken.

\section{Disclosure}

The authors report no conflict of interest concerning the materials or methods used in this study or the findings specified in this paper.

Author contributions to the study and manuscript preparation include the following. Conception and design: Ius, Skrap. Acquisition of data: Ius. Analysis and interpretation of data: Ius, Isola, Skrap. Drafting the article: Ius, Isola, Skrap. Critically revising the article: all authors. Reviewed submitted version of manuscript: all authors. Approved the final version of the manuscript on behalf of all authors: Ius. Statistical analysis: Ius, Isola. Administrative/technical/ material support: Ius. Study supervision: Ius, Skrap.

\section{Acknowledgment}

The authors thank Dr. Jonathan Cook (Advance Consulting) for valuable advice in proofreading.

\section{References}

1. Ahmadi R, Dictus C, Hartmann C, Zürn O, Edler L, Hartmann $\mathrm{M}$, et al: Long-term outcome and survival of surgically treated supratentorial low-grade glioma in adult patients. Acta Neurochir (Wien) 151:1359-1365, 2009 (Erratum in Acta Neurochir (Wien) 15:1367, 2009)

2. Bauman G, Lote K, Larson D, Stalpers L, Leighton C, Fisher B, et al: Pretreatment factors predict overall survival for patients with low-grade glioma: a recursive partitioning analysis. Int J Radiat Oncol Biol Phys 45:923-929, 1999

3. Bello L, Castellano A, Fava E, Casaceli G, Riva M, Scotti G, et al: Intraoperative use of diffusion tensor imaging fiber tractography and subcortical mapping for resection of gliomas: technical considerations. Neurosurg Focus 28(2):E6, 2010 
4. Berger MS: Functional mapping-guided resection of lowgrade gliomas. Clin Neurosurg 42:437-452, 1995

5. Berger MS, Deliganis AV, Dobbins J, Keles GE: The effect of extent of resection on recurrence in patients with low grade cerebral hemisphere gliomas. Cancer 74:1784-1791, 1994

6. Berger MS, Ojemann GA: Intraoperative brain mapping techniques in neuro-oncology. Stereotact Funct Neurosurg 58: 153-161, 1992

7. Berger MS, Rostomily RC: Low grade gliomas: functional mapping resection strategies, extent of resection, and outcome. J Neurooncol 34:85-101, 1997

8. Berman J: Diffusion MR tractography as a tool for surgical planning. Magn Reson Imaging Clin N Am 17:205-214, 2009

9. Berman JI, Berger MS, Chung SW, Nagarajan SS, Henry RG: Accuracy of diffusion tensor magnetic resonance imaging tractography assessed using intraoperative subcortical stimulation mapping and magnetic source imaging. J Neurosurg 107: 488-494, 2007

10. Bertani G, Fava E, Casaceli G, Carrabba G, Casarotti A, Papagno $\mathrm{C}$, et al: Intraoperative mapping and monitoring of brain functions for the resection of low-grade gliomas: technical considerations. Neurosurg Focus 27(4):E4, 2009

11. Black PM, Ronner SF: Cortical mapping for defining the limits of tumor resection. Neurosurgery 20:914-919, 1987

12. Blonski M, Taillandier L, Herbet G, Maldonado IL, Beauchesne P, Fabbro M, et al: Combination of neoadjuvant chemotherapy followed by surgical resection as a new strategy for WHO grade II gliomas: a study of cognitive status and quality of life. J Neurooncol 106:353-366, 2012

13. Bourne TD, Schiff D: Update on molecular findings, management and outcome in low-grade gliomas. Nat Rev Neurol 6: 695-701, 2010

14. Byrne TN: Response of low-grade oligodendroglial tumors to temozolomide. J Neurooncol 70:279-280, 2004

15. Chaichana KL, McGirt MJ, Laterra J, Olivi A, Quiñones-Hinojosa A: Recurrence and malignant degeneration after resection of adult hemispheric low-grade gliomas. Clinical article. J Neurosurg 112:10-17, 2010

16. Claus EB, Horlacher A, Hsu L, Schwartz RB, Dello-Iacono D, Talos F, et al: Survival rates in patients with low-grade glioma after intraoperative magnetic resonance image guidance. Cancer 103:1227-1233, 2005

17. Duffau H: Lessons from brain mapping in surgery for lowgrade glioma: insights into associations between tumour and brain plasticity. Lancet Neurol 4:476-486, 2005

18. Duffau H: New concepts in surgery of WHO grade II gliomas: functional brain mapping, connectionism and plasticity-a review. J Neurooncol 79:77-115, 2006

19. Duffau H: Surgery of low-grade gliomas: towards a 'functional neurooncology.' Curr Opin Oncol 21:543-549, 2009

20. Duffau H, Capelle L: Preferential brain locations of low-grade gliomas. Cancer 100:2622-2626, 2004

21. Duffau H, Capelle L, Sichez N, Denvil D, Lopes M, Sichez JP, et al: Intraoperative mapping of the subcortical language pathways using direct stimulations. An anatomo-functional study. Brain 125:199-214, 2002

22. Duffau H, Lopes M, Arthuis F, Bitar A, Sichez JP, Van Effenterre R, et al: Contribution of intraoperative electrical stimulations in surgery of low grade gliomas: a comparative study between two series without (1985-96) and with (1996-2003) functional mapping in the same institution. J Neurol Neurosurg Psychiatry 76:845-851, 2005

23. Grier JT, Batchelor T: Low-grade gliomas in adults. Oncologist 11:681-693, 2006

24. Ius T, Angelini E, Thiebaut de Schotten M, Mandonnet E, Duffau $\mathrm{H}$ : Evidence for potentials and limitations of brain plasticity using an atlas of functional resectability of WHO grade II gliomas: towards a "minimal common brain." Neuroimage 56:992-1000, 2011
25. Jansen M, Yip S, Louis DN: Molecular pathology in adult gliomas: diagnostic, prognostic, and predictive markers. Lancet Neurol 9:717-726, 2010

26. Jenkins RB, Blair H, Ballman KV, Giannini C, Arusell RM, Law M, et al: A $\mathrm{t}(1 ; 19)(\mathrm{q} 10 ; \mathrm{p} 10)$ mediates the combined deletions of $1 \mathrm{p}$ and $19 \mathrm{q}$ and predicts a better prognosis of patients with oligodendroglioma. Cancer Res 66:9852-9861, 2006

27. Johannesen TB, Langmark F, Lote K: Progress in long-term survival in adult patients with supratentorial low-grade gliomas: a population-based study of 993 patients in whom tumors were diagnosed between 1970 and 1993. J Neurosurg 99:854862,2003

28. Keles GE, Lamborn KR, Berger MS: Low-grade hemispheric gliomas in adults: a critical review of extent of resection as a factor influencing outcome. J Neurosurg 95:735-745, 2001

29. Kinoshita M, Yamada K, Hashimoto N, Kato A, Izumoto S, Baba T, et al: Fiber-tracking does not accurately estimate size of fiber bundle in pathological condition: initial neurosurgical experience using neuronavigation and subcortical white matter stimulation. Neuroimage 25:424-429, 2005

30. Lang FF, Olansen NE, DeMonte F, Gokaslan ZL, Holland EC, Kalhorn C, et al: Surgical resection of intrinsic insular tumors: complication avoidance. J Neurosurg 95:638-650, 2001

31. Laws ER Jr, Taylor WF, Clifton MB, Okazaki H: Neurosurgical management of low-grade astrocytoma of the cerebral hemispheres. J Neurosurg 61:665-673, 1984

32. Leighton C, Fisher B, Bauman G, Depiero S, Stitt L, MacDonald D, et al: Supratentorial low-grade glioma in adults: an analysis of prognostic factors and timing of radiation. J Clin Oncol 15:1294-1301, 1997

33. Louis DN, Ohgaki H, Wiestler OD, Cavenee WK, Burger PC, Jouvet A, et al: The 2007 WHO classification of tumours of the central nervous system. Acta Neuropathol 114:97-109, 2007

34. Mandonnet E, Capelle L, Duffau H: Extension of paralimbic low grade gliomas: toward an anatomical classification based on white matter invasion patterns. J Neurooncol 78:179-185, 2006

35. McGirt MJ, Chaichana KL, Attenello FJ, Weingart JD, Than K, Burger PC, et al: Extent of surgical resection is independently associated with survival in patients with hemispheric infiltrating low-grade gliomas. Neurosurgery 63:700-708, 2008

36. McGirt MJ, Chaichana KL, Gathinji M, Attenello FJ, Than K, Olivi A, et al: Independent association of extent of resection with survival in patients with malignant brain astrocytoma. Clinical article. J Neurosurg 110:156-162, 2009

37. Mori S, van Zijl PC: Fiber tracking: principles and strategiesa technical review. NMR Biomed 15:468-480, 2002

38. Nakamura M, Konishi N, Tsunoda S, Nakase H, Tsuzuki T, Aoki $\mathrm{H}$, et al: Analysis of prognostic and survival factors related to treatment of low-grade astrocytomas in adults. Oncology 58:108-116, 2000

39. North CA, North RB, Epstein JA, Piantadosi S, Wharam MD: Low-grade cerebral astrocytomas. Survival and quality of life after radiation therapy. Cancer 66:6-14, 1990

40. Ojemann G, Ojemann J, Lettich E, Berger M: Cortical language localization in left, dominant hemisphere. An electrical stimulation mapping investigation in 117 patients. J Neurosurg 71:316-326, 1989

41. Oldfield RC: The assessment and analysis of handedness: the Edinburgh inventory. Neuropsychologia 9:97-113, 1971

42. Philippon JH, Clemenceau SH, Fauchon FH, Foncin JF: Supratentorial low-grade astrocytomas in adults. Neurosurgery 32:554-559, 1993

43. Rajan B, Pickuth D, Ashley S, Traish D, Monro P, Elyan S, et al: The management of histologically unverified presumed cerebral gliomas with radiotherapy. Int J Radiat Oncol Biol Phys 28:405-413, 1994

44. Rosset A, Spadola L, Ratib O: OsiriX: an open-source software for navigating in multidimensional DICOM images. J Digit Imaging 17:205-216, 2004 
45. Rostomily RC, Keles GE, Berger MS: Radical surgery in the management of low-grade and high-grade gliomas. Baillieres Clin Neurol 5:345-369, 1996

46. Sanai N, Berger MS: Glioma extent of resection and its impact on patient outcome. Neurosurgery 62:753-764, 2008

47. Sanai N, Berger MS: Operative techniques for gliomas and the value of extent of resection. Neurotherapeutics 6:478-486, 2009

48. Sanai N, Chang S, Berger MS: Low-grade gliomas in adults. A review. J Neurosurg 115:948-965, 2011

49. Sanai N, Polley MY, Berger MS: Insular glioma resection: assessment of patient morbidity, survival, and tumor progression. Clinical article. J Neurosurg 112:1-9, 2010

50. Shaw E, Arusell R, Scheithauer B, O'Fallon J, O’Neill B, Dinapoli R, et al: Prospective randomized trial of low- versus high-dose radiation therapy in adults with supratentorial lowgrade glioma: initial report of a North Central Cancer Treatment Group/Radiation Therapy Oncology Group/Eastern Cooperative Oncology Group study. J Clin Oncol 20:22672276, 2002

51. Simon M, Neuloh G, von Lehe M, Meyer B, Schramm J: Insular gliomas: the case for surgical management. Clinical article. J Neurosurg 110:685-695, 2009

52. Skrap M, Mondani M, Tomasino B, Weis L, Budai R, Pauletto $\mathrm{G}$, et al: Surgery of insular nonenhancing gliomas: volumetric analysis of tumoral resection, clinical outcome, and survival in a consecutive series of 66 cases. Neurosurgery 70:10811094, 2012

53. Smith JS, Cha S, Mayo MC, McDermott MW, Parsa AT, Chang SM, et al: Serial diffusion-weighted magnetic resonance imaging in cases of glioma: distinguishing tumor recurrence from postresection injury. J Neurosurg 103:428-438, 2005

54. Smith JS, Chang EF, Lamborn KR, Chang SM, Prados MD, Cha $\mathrm{S}$, et al: Role of extent of resection in the long-term outcome of low-grade hemispheric gliomas. J Clin Oncol 26:1338-1345, 2008

55. Soffietti R, Baumert BG, Bello L, von Deimling A, Duffau
H, Frénay M, et al: Guidelines on management of low-grade gliomas: report of an EFNS-EANO Task Force. Eur J Neurol 17:1124-1133, 2010

56. Stadlbauer A, Nimsky C, Buslei R, Salomonowitz E, Hammen $\mathrm{T}$, Buchfelder M, et al: Diffusion tensor imaging and optimized fiber tracking in glioma patients: histopathologic evaluation of tumor-invaded white matter structures. Neuroimage 34:949956, 2007

57. Steyerberg EW: Clinical Prediction Models: A Practical Approach to Development, Validation, and Updating. New York: Springer, 2009

58. van Veelen ML, Avezaat CJ, Kros JM, van Putten W, Vecht C: Supratentorial low grade astrocytoma: prognostic factors, dedifferentiation, and the issue of early versus late surgery. J Neurol Neurosurg Psychiatry 64:581-587, 1998

59. Wessels PH, Weber WE, Raven G, Ramaekers FC, Hopman AH, Twijnstra A: Supratentorial grade II astrocytoma: biological features and clinical course. Lancet Neurol 2:395-403, 2003

60. Whitton AC, Bloom HJ: Low grade glioma of the cerebral hemispheres in adults: a retrospective analysis of 88 cases. Int J Radiat Oncol Biol Phys 18:783-786, 1990

61. Yeh SA, Ho JT, Lui CC, Huang YJ, Hsiung CY, Huang EY: Treatment outcomes and prognostic factors in patients with supratentorial low-grade gliomas. Br J Radiol 78:230-235, 2005

Manuscript submitted February 17, 2012.

Accepted August 29, 2012.

Please include this information when citing this paper: published online October 5, 2012; DOI: 10.3171/2012.8.JNS12393.

Address correspondence to: Tamara Ius, M.D., Department of Neurosurgery, Azienda Ospedaliero-Universitaria Santa Maria della Misericordia, Piazzale Santa Maria della Misericordia 15, 33100 Udine, Italy.email: tamara.ius@gmail.com. 